\title{
16 Conclusion
}

\section{The ambiguity of cyber security politics in the context of multidimensional uncertainty}

\author{
Andreas Wenger and Myriam Dunn Cavelty
}

In a world of rapid socio-technical transformation and increasing fragmentation of political power and authority, cyber security has firmly established itself as one of the top national security issues of the 21 st century. Managing cyber insecurities will most likely further increase in complexity and political significance in the next decade, co-produced by an acceleration of the ongoing socio-technical transformations, on the one hand, and the changing dynamics of the related political responses, on the other. The first part of the book recorded the ongoing geographic expansion of cyberspace into outer space, anticipated how emerging technologies will increase the interconnectedness of infrastructures and services, and projected how in a context of ever tighter coupled and integrated socio-technical systems cyber threat narratives will inevitably expand to more policy fields at both the national and international levels. The second part of the book discussed how in cyberspace state actors need to find the right balance between restraint and exploitation, why they need to uphold their efforts to control the risk of escalation, and why governments increasingly share responsibility with actors from economy and society.

The current state of cyber security politics is very much a reflection of the interplay between the underlying forces of great power competition and the dynamics of socio-technical and socio-economic globalization processes. From the interplay of these two processes emerge the two key factors - multidimensional uncertainty and socio-political ambiguity - that characterize the current context of cyber security politics at both the national and international levels, as highlighted in Figure 16.1. Multidimensional uncertainty plays a key role in the emergence of cyber insecurity as a wicked problem and shapes - and is shaped by - the ambiguity of cyber security politics.

The ambiguity of cyber security politics encompasses the two dimensions of cyber security outlined in the introductory chapter (Dunn Cavelty and Wenger 2022): First, the international dimension of cyber security politics concentrates on how state actors shape and use cyberspace in accordance with their strategic goals, while at the same time struggling to uphold the stability of their strategic relationships. In Figure 16.1, the interactive search for an acceptable balance between 


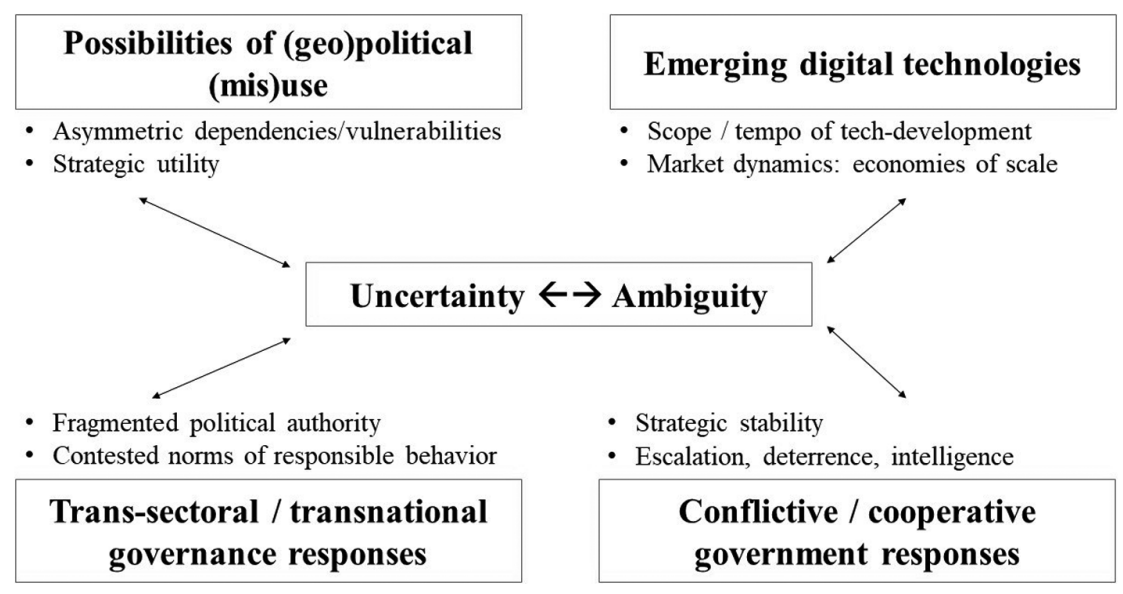

Figure 16.1 The dimensions of cyber security.

the strategic utility of and the strategic stability in cyberspace is represented in the upper left (possibilities of (geo)political (mis)use) and lower right (conflictive / cooperative government responses) corners. Second, the broader dimension of cyber security politics focuses on how state, industry, and societies negotiate their respective roles in governing cyberspace, while at the same time competing in the tech innovation process that affects the continued transformation of cyberspace. In Figure 16.1, the interactive search for norms of responsible behavior in an uncertain and ambiguous socio-technical and sociopolitical context is represented in the lower left (fragmented trans-sectoral/transnational governance responses) and upper right (emerging digital technologies) corners.

This concluding chapter, building on the individual contributions to this book, highlights four key debates that together encapsulate the complexities and paradoxes of the current thinking about the future of cyber security politics from a Western perspective. The first section asks how much political influence states can achieve via cyber operations and what context factors condition the (limited) strategic utility of such operations. A second section discusses the role of emerging digital technologies in cyber security politics and notes how the dynamics of the tech innovation process reinforce the fragmentation of the governance space around them. A third section asks how states attempt to uphold stability in cyberspace, and in their strategic relations more general, highlighting three interconnected challenges - escalation, deterrence, and intelligence - of this interactive quest. A fourth and final section focuses on the shared responsibility of state, economy, and society for cyber security and calls attention to the continuing renegotiation processes about their respective roles in an increasingly trans-sectoral and transnational governance space. 


\section{The strategic utility of cyber operations}

The debate about the strategic utility of cyber operations arises in a context characterized by the interplay between the rapid emergence of new digital technologies and the politics of their use and misuse. Over time, the debate evolved considerably, as cyber security issues transformed from a technical risk management issue discussed by a limited circle of experts into a key challenge of national security debated at the highest level of governments (Dunn Cavelty 2008; Dewar 2018). In its early stages, the debate focused on "doomsday" cyberattack scenarios that centered on the strategic exploitation of increasingly interconnected and vulnerable infrastructures (Clarke and Knake 2010). As out-of-the-blue cyber war failed to make its expected appearance, experts began to shift their attention to the political and strategic implications of low-level cyber conflict (Baezner 2018; see also Rid 2012; Lindsay 2014/15), on the one hand, and to the increase of computer network attack campaigns linked to covert state involvement (Dunn Cavelty 2015), on the other.

At the current point in time in the history of cyber security politics the empirical picture is characterized by "dogs that did not bark" at the high end of conflict and persistent cyber operations and instability at the low end of conflict (Schulze 2020; Harknett and Smeets 2020; Lupovici 2021). Within this context, the chapters in this volume point to three interconnected aspects of the enduring debate about the strategic utility of cyber operations: A first subsection concentrates on the difficulty of achieving a controlled strategic effect under multidimensional uncertainty. The focus here is on explaining why most cyber operations so far seem not very escalatory and appear unlikely to result in visible changes in the existing balance of power between great powers. A second subsection focuses on the utility of cyber operations as a tool of subversion and mild sabotage. Here the focus is on understanding how the ambiguity of involved actors and the opaqueness of cyber operations can be manipulated in specific strategic contexts by some powers for asymmetric influence. A third subsection deals with the assumed asymmetrical vulnerability of democracies to disinformation as the latest cyber threat focus in Western (security) politics. Here the debate centers on the question if a strategic effect can be achieved via cyber influence operations that aim at undermining social cohesion and trust in democratic political institutions.

\section{The difficulty of achieving a controlled strategic effect under multidimensional uncertainty}

Several chapters in this volume engage with the notion that cyber operations are of limited strategic utility in terms of transforming the balance of economic and military power at the level of interstate relations or, more specifically, in terms of an adversary changing its rival's political goals (Gomez and Whyte 2022; Baezner and Cordey 2022). The authors do not explicitly dispute the conclusion of the strategic studies literature that a strategic impact of cyber operations might be elusive (Smeets 2018; Borghard and Lonergan 2017: 477; Kostyuk and Zhukov 2017; 
Valeriano and Maness 2015: 183; Gartzke 2013). Yet they are concerned, albeit for different reasons, that the insights of this literature might translate into policies that underestimate the escalatory risk of persistent engagement and defend forward (see also Devanny 2021; Healey and Jervis 2020; Healey 2019; Cavaiola et al. 2015). We will come back to the problem of upholding strategic stability under multidimensional uncertainty below.

Operating strategically in cyberspace, so much seems to be clear, is far more technically and operationally demanding than the "cheap and easy" metaphor suggests (Lindsay 2013; Slayton 2017; Lewis 2018). "Causing a specific, targeted cyber effect, at a designated point in time, which achieves a strategic purpose, and outweighs the impact of negative consequences, is hard", Max Smeets notes in a forthcoming book (Smeets forthcoming). Resources constrain the overall utility expected from cyber operations. This is a point reinforced by the economic logic of cyber influence, as Jon R. Lindsay has argued (Lindsay 2017). He holds that setting up cyber exploitations is generally more expensive than countering them, which increases the incentive to keep the target at risk over longer periods of time, turning cyber conflict into primarily an intelligence game (cf. Chesney and Smeets forthcoming; Chesney and Smeets 2020; Rovner 2019). These techni$\mathrm{cal}$, organizational, and economic challenges all reflect the structural features of cyberspace.

Achieving a controlled strategic impact via cyber operations is challenging because cyberspace as an operating environment is characterized by multidimensional uncertainty and sociopolitical ambiguity. On the one hand, cyberspace is marked by a high degree of interconnectedness. This very feature makes it very difficult to fully control the strategic effects of cyber operations, since some unintended side-effects in the sense of collateral damage beyond the intended target seem almost unavoidable (Smeets 2018). On the other hand, cyberspace is characterized by constant political contestation. This makes it very difficult to achieve a stable political outcome in which an adversary changes their political goals. According to the same logic, attribution of cyber operations to specific political actors remains time-consuming and often inconclusive (Rid and Buchanan 2015). Neither states nor cyber intelligence firms have enough of an incentive to fully share the data, methods, and tools behind their attribution claims (Egloff 2020a, Egloff and Wenger 2019). As a consequence, many attribution processes lack transparency and credibility, making it difficult to build broad and stable political support for response strategies based on inherently contested attribution claims (Egloff and Dunn Cavelty 2021).

In the context of political competition, cyber operations lack strategic utility as a stand-alone tool to gain an enduring political or military advantage. In actual practice, however, they are linked to and integrated with a broad range of other foreign and security policy instruments. The covert nature of cyber operations means that elites use them as instruments that signal resolve while minimizing escalation risks (Poznansky and Perkoski 2018). The second subsection turns to the question how certain actors attempt to manipulate cyber operations in certain strategic contexts for limited asymmetric influence. 


\section{The power to subvert: manipulating "gray zones" while minimizing the risk of escalation}

Most cyber operations take place below the threshold of war, Marie Baezner and Sean Cordey (2022) note in their chapter. Mapping the practical use of such operations in a series of cyber conflict case studies, they confirm that especially influence operations fall into a zone which goes beyond conventional diplomacy and stops short of conventional war, which Lucas Kello describes as "unpeace" (Kello 2017). Taking this empirical puzzle as a starting point for their analysis, the chapter asks why some actors see such operations as attractive and efficient tools of power projection and influence. The (limited) strategic utility of cyber (influence) operations, the two authors conclude, depends on the characteristics of the strategic context and the operational environment in which they are employed and on the nature of the strategic actor employing such operations.

At a strategic level, the increasingly pervasive use of cyber (influence) operations in international affairs reflects the current dynamics of great power competition. Together, the increasing costs of conventional war and the realities of economic interdependence create incentives, especially for great powers, to gain asymmetric influence through cyber operations, in particular in their spheres of interest, without however unduly undermining the strategic stability of great power relations. At an operational level, the use of cyber influence operations reflects an operational environment that is characterized by legal ambiguity and political contestation, opacity of the parties involved and blurred boundaries between the private and public domains. Referring to the concept of and literature on "gray zones", Baezner and Cordey argue that revisionist powers use cyber operations as tools to operate below the threshold of armed combat to gain an asymmetric advantage in their relationship with other political actors, especially in view of the global (military) dominance of the United States.

Based on a series of case studies, Baezner and Cordey note that the following operational assumptions about cyber (influence) operations seem to make them attractive tools for many to intervene in gray zone conflicts. First, the majority of the cyber technologies used in such contexts are widely available at relatively low cost. Patriotic hackers or opaque criminal groups with ties to domestic or foreign elites use them opportunistically for disruption and mild sabotage rather than for destruction. Second, cyber espionage and influence operations are increasingly used to influence the information environment of a conflict and gain an asymmetric advantage. They work in tandem with a wider set of economic, political, and military coercive tools. Third, the legal uncertainty surrounding intelligence operations allows state actors to avoid formal condemnation and uphold a posture of plausible deniability. The opaqueness of actors and operations makes it unlikely that a verdict of attribution would be as transparent and credible as to justify a military response.

The importance of the strategic context and the nature of the strategic actor employing cyber (influence) operations are confirmed by Aaron Brantly (2022) in his chapter on Ukraine. He analyzes Ukraine as a case of how to confront a larger 
aggressive adversary employing cyber and information warfare in its considered sphere of influence at a time of extreme domestic vulnerability amid violent regime change. Before the 2014 Euromaidan revolution, widespread rent-seeking behavior of criminal-political patronage networks and extensive penetration of Ukraine's state structures by Russia's intelligence service made Ukraine vulnerable to foreign cyber and influence operations. The revolution reversed Ukraine's foreign policy alignment from Russia to the West and began a slow process of domestic legal, organizational, and policy transformation that however remains contested by entrenched elites. Both the relative success of Russia's cyber and information warfare as well as the relative success of Ukraine's response to Russian cyberattacks and disinformation campaigns, Brantly notes, must be assessed in the context of broader patterns of domestic political contestation, on the one hand, and the countries' international orientation and dependence, on the other.

Cyber operations in "gray zone" strategic contexts should be conceptualized less as a means of warfare and more appropriately as a tool of political power projection, Marie Baezner and Sean Cordey conclude. The two authors see such operations as both a novel, efficient and effective tool for disruption (and, to a lesser extent, sabotage) and an "enhancer and transformer" of traditional espionage and covert intelligence operations (Baezner and Cordey 2022: 25). Although their strategic utility will remain elusive, they argue, actors operating in the "gray zones" of modern conflict will likely continue to invest into cyber operations and use them in order to gain an asymmetric advantage. Yet the widely shared assumption that cyber (influence) operations carry a limited risk of escalation might be misplaced and should be reconsidered, the two authors argue. As long as there is a lack of consensus among great powers about norms of acceptable espionage and as long as their definitions of cyber security diverge, the risks of unintentional escalation remain worrisome.

\section{Disinformation as a new threat focus: Asymmetrical vulnerability of democracies?}

In cyberspace "the power to subvert seems to trump both the power to coerce and the power to attract" (Dunn Cavelty and Wenger 2019: 15). Subversive power is especially relevant in strategic contexts in which the perceived spheres of interest by rising powers overlap with the geopolitical interests of ruling powers that uphold the status quo (Maschmeyer 2021). But to what degree can revisionist powers use cyber influence operations also as effective tools to undermine the social cohesion and the political stability of democracies? This concern has turned into one of the most relevant cyber threat narratives in Western policy circles, ever since US authorities have attributed the cyber campaigns targeting the US election in 2016 to Russia (Egloff 2020b).

Western policymakers increasingly perceive disinformation and cyber influence campaigns by Russia and China as a major threat to liberal democracies, Wolf J. Schünemann (2022) notes in his chapter. Analyzing the threat frames used 
in Western policy documents, he shows that Western policymakers conceptualize disinformation campaigns that target democratic elections as strategic tools used by Russia and China in the context of great power competition. According to the threat narrative that emerges from these policy documents, foreign actors are actively exploiting the bias of liberal democracies against media control. They actively manipulate the ambiguities between public diplomacy and coordinated disinformation campaigns to target and potentially distort national elections. Such a threat narrative is often connected to a policy response that aims at strengthening the state's strategic communication capacities. This, however, Schünemann cautions, might have unintended side-effects. Expanding the control of the state over the information sphere might weaken a democracy's best barriers against disinformation: public discourse and public opinion.

The political context of the alleged asymmetrical vulnerability of democracies is characterized by uncertainty and a lack of knowledge about the actual impact of disinformation. There is very little robust empirical evidence, Schünemann notes, that foreign disinformation campaigns have a substantial long-term effect on public discourse and public policy. The potential macro effects on political discourses are very difficult to understand and to prove, not least because the digital public sphere and the mass media system are themselves in the middle of a structural transformation. Several phenomena associated with this transformation - for example, echo chambers and automated social bots - are seen as facilitating factors for the spread of disinformation. Yet there is little robust evidence about how they influence the processes of political opinion formation at the macro level (also see Maschmeyer et al. forthcoming). Understanding how the attack surface - the public sphere and public discourse - is changing in the context of digitalization is a precursor for the study of the impact that disinformation might have on political discourse and electoral processes at the national level.

New digital tools such as social media have a potential - with or without outside interference - to erode social trust and increase political fragmentation in (democratic) societies. The use of new socio-technical tools, however, is not predetermined, as Jasmin Haunschild, Marc-André Kaufhold, and Christian Reuter demonstrate in their chapter (Haunschild et al. 2022). This means, they argue, that new socio-technical countermeasures can be designed and developed that ameliorate the potentially negative effects of social and political bots. New technologies can be used to increase social cohesion or to exploit existing grievances. And while tech race dynamics can be strong - for example, between automated bot configuration and automated bot detection - social intervention will remain decisive. Their chapter highlights that the micro-politics of business and civilian actors designing the right social-technical tools might be as important for the resilience of democratic societies against disinformation campaign as the macropolitical responses of state (security) organizations.

The effectiveness of foreign disinformation and propaganda is linked to the exploitation of preexisting social distrust and political grievances. On this, the authors of Chapters 3 and 4 agree. Successful disinformation campaigns exploit existing vulnerabilities of the public discourse and as such must be reduced from 
within, Schünemann notes. Uncertainty about the potential negative effect of foreign disinformation, he concludes, "must not let us stumble into a new phase of international threat politics and of securitisation of cyberspace with potentially detrimental effects on liberal democratic values and international peace" (Schünemann 2022: 33). Increasing the resilience of democratic societies against foreign disinformation campaigns remains a shared responsibility of civil society, the private and the public sectors.

\section{Emerging technologies and the future of cyber security politics}

Ever since cyber security issues have appeared on the agenda of national and international politics, Jon R. Lindsay (2022) argues in his chapter on the ambiguity of a cryptologic advantage, two analytically distinct perspectives have informed the debate about their relevance for cyber security politics. A first perspective builds on the premise that technology determines politics. Anticipating the transformative potential of emerging technologies, this view tends to extrapolate dramatic consequence for security politics. We have already reviewed early expert assumptions along the line that the nature of cyberspace is destabilizing and favors the offense. A second perspective starts from the opposite end of the relationship and assumes that politics determines technology. Such an analytical perspective translates into expectations that the sociopolitical context mitigates the supposed advantages of cyber offense and reinforces established power relationships (cf. Dunn Cavelty and Wenger 2019).

We argue throughout the volume that a perspective that combines the two views and unpacks the co-constitution and co-dependency of technology and politics provides a more productive analytical lens for studying the ambiguous implications of rapid technological change on cyber security politics and vice versa. Within this context, the chapters in this volume discuss three key insights on the interrelationship between emerging technologies and the future of cyber security politics. A first subsection concentrates on tech race dynamics as drivers of cyber threat perceptions. The focus here is on the interplay between global market and geopolitical dynamics under multidimensional uncertainty and how these dynamics feed into threat narratives. A second subsection highlights that the sociopolitical context conditions the strategic utility of emerging technologies. Here the focus is on how social and institutional factors shape the influence that emerging technologies have on the balance between the offense and the defense. A third subsection deals with the growing role of private actors in digital innovation in general and in securing cyberspace more specifically. The focus here is on how the multiplication of actors increases the socio-technical uncertainty and the sociopolitical ambiguity of the governance space around emerging technologies.

\section{Tech race dynamics as drives of cyber threat perceptions}

The dynamic and emergent trajectory of technology development is a key factor shaping the interplay between technology and politics. Multidimensional 
uncertainty - about the scope and tempo of the technological development and about market dynamics and social acceptance - is a key driver of the innovation process (see Figure 16.1). Technology firms are exposed to market pressures and driven by profit. They make their design and development decisions, including complex trade-offs between the performance and the safety and transparency of their products and services, in the shadow of a potential first-mover advantage and the promise of huge economies of scale. Conversely, governments influence the innovation process via the formulation and implementation of technology strategies that specify national levels of ambition. Such strategies aim at incentivizing the domestic uptake of new technologies and creating a regulatory environment that fits their societies' institutional and normative contexts while positioning their countries in the best possible way in the emerging global innovation space (Bonfanti 2022).

As new technological possibilities - linked to the development of artificial intelligence, quantum computing, or space technologies - appear on the horizon, both governments and corporations focus on their potentially transformative capacities, and, more specifically, anticipate what role these technologies will play in shaping cyber security. Most technologies discussed in the chapters of this volume are dualuse technologies and as such might influence the global economic and military balance. As a consequence, great powers tend to treat such technologies as a potential strategic resource. Out of these technical, economic, and (geo)political dynamics an ambiguous political interaction dynamic evolves that fits the logic of the security dilemma (Jervis 1978): The means - in this case maneuvring to attain or sustain an advantage in critical technologies - by which a state tries to maximize its national interests and security threatens the interests and relative security of other states.

From a political perspective, it is problematic if the technology development process is dominated by only a few dominant economic (global tech firms) and political actors (great powers). A concentration of technical resources in the hands of a few actors might affect the global distribution of economic and military power and create or deepen asymmetric economic and political dependencies. A context of an intensifying technology competition creates incentives for states to influence the innovation process and the proliferation of new technology in their narrow national interest (Fischer and Wenger 2019). Conversely, technology race dynamics act as drivers of national threat perceptions and tend to feed doom scenarios. State actors see themselves increasingly caught in a global race for AI or quantum dominance (Lindsay 2022; Bonfanti 2022). From the perspective of science and technology studies, such threat narratives are co-constituted by the micro-politics of design decisions in competitive global markets and the macropolitics of great powers that act strategically in a competitive international system (Fischer and Wenger 2021).

\section{The strategic utility of emerging technologies depends on the sociopolitical context}

The insight that the balance between offense and defense in intelligence has always depended more on institutional factors and strategic context than on technological 
architecture represents the key message of Chapter 6 in this volume. Analyzing the tumultuous relationship between cryptologic technology and political advantage, Jon R. Lindsay (2022) highlights the fundamental political paradox between cryptography (code-making) and cryptanalysis (code-breaking): They must cooperate to compete and respect the constraints of a cooperatively produced cryptosystem. As a consequence, cryptology turns into an organizational contest and as such heavily depends on social factors. It does not come as a surprise against this background that one of the central insights of cryptologic history is that "gullible humans are the Achilles Heel of classical cryptology" (Lindsay 2022: 89). This again, Lindsay argues, makes it reasonable to expect that humans "will also be the undoing of quantum cryptology" (Lindsay 2022: 89).

A working quantum computer should be able to crack the current cryptographic protocols that are vital for cyber security. Anticipating a one-sided technological breakthrough easily translates into fear that a breakthrough in quantum computing might compromise the existing public key infrastructure. As China began to heavily invest into quantum technology, a threat narrative evolved in Western states that perceived the great powers to be locked into a global race to gain a quantum advantage. A quantum breakthrough would have major repercussion for security and defense, so the arguments went, since one's own intelligence would be locked out while the first-movers' communication would become impenetrable. Should this indeed happen, policymakers and strategists feared, global stability could be at risk.

Yet the implications of the interaction between technology and politics will likely be more ambiguous, Lindsay argues. First, such a perspective overlooks that the search for quantum safe protocols begins parallel to the development of a quantum computer that would be able to break the current cryptographic protocols. Second, quantum computing would not change the reliance of cryptology on social factors. Intelligence remains fundamentally a contest between human organizations. The current golden age of cyber espionage was not enabled by a mathematically and technically weak public key infrastructure. It can be traced back to an overly complex organizational setup of the infrastructure and poor cyber hygiene among computer users. Third, even if one side in a geopolitical contest would develop a cryptographic advantage, how this advantage would translate into a political outcome is not predetermined by technology. Rather it would be contingent on the overarching strategic context and the specifics of institutional decision-making. A cryptanalytic success, Lindsay notes, can make a bargain more likely or a surprise attack more attractive, and it may even provide a false sense of security.

In his chapter, Matteo E. Bonfanti (2022) in a similar vein discusses the implications of emerging AI technologies for the offense-defense balance in cyber security. These implications are difficult to predict, he argues, because the context is characterized by widespread uncertainty and ambiguity. Most AI tools can be used in support of both cyber defense as well as cyber offense. AI-based cyber capabilities will affect both the logical (software) dimension as well as the semantic (content) dimension of cyberspace. That AI will have major implications for 
cyber security is undisputed among experts. Yet who will be the winner - offense or defense, states security agencies or private threat intelligence firms, democracies or autocracies - remains to be seen. The eventual outcome of the integration of AI technologies into cyber security depends on the strategic and sociopolitical context and the risk-benefit calculations of many different public and private cyber security stakeholder.

\section{Private actor innovation increases socio-technical uncertainty and sociopolitical ambiguity}

The growing role of private actors in cyber security and in the digital innovation process is noted in most chapters of the book. Big technology companies make key contributions to the development and operation of cyberspace. Private companies act as operators of networks, designers of products and suppliers of services (Eggenschwiler 2022). Small and large technology companies drive the AI innovation process (Bonfanti 2022). Private actors make smaller and more efficient satellites and have turned into key players in the integration of cyberspace and outer space. The growing role of private actors in outer space was enabled by legal changes in the United States and other states that in the context of a neoliberal vision of state-business relationship opened dual-use space projects to private investment and research and development (Erikkson and Giacomello 2022).

Over the past 30 years, the global technology innovation system has increasingly been shaped by the twin forces of globalization and commercialization. While during the Cold War, the development of nuclear, chemical, and biological dual-use technologies was dominated by state investment and national security concerns, the tide began to turn toward a private sector lead as the development of digital technologies began to take off during the 1990s when the first mobile phones and the internet were made available to the broader public (Fischer 2021). The multiplication of actors in digital innovation and cyber security had ambiguous implications, as the incentive structure of widely heterogonous and increasingly transnationally active technology companies increased the prevailing socio-technical uncertainties. Private technology firms are primarily driven by profit and economies of scale. Although their collective business success depends on high levels of social trust in digital technologies and infrastructures, individual firms have a structural motivation to protect their trade secrets and not to fully disclose all their data and algorithms.

Private actors are not only a key innovator of digital technologies, but they have also dramatically expanded their role in securing cyberspace. Brenden Kuerbis, Farzaneh Badiei, Karl Grindal, and Mitlon Mueller (2022) show in their chapter that private threat intelligence firms have turned into key attribution actors. The forensic capabilities of some of the bigger transnational firms are more advanced than those of many states. Yet their attribution claims lack transparency and public legitimacy (Egloff and Wenger 2019). Moreover, it is unclear why they would contribute to a transnational attribution authority, Kuerbis et al. (2022) note. Conversely, the average dependence of critical public security services on private technology companies 
providing specialized services in the area of big data analytics and AI-based automated evaluation and assessment will likely grow in the future. Already today, technology consultancy firms provide critical services for states' intelligence services, military (cyber) commands, and national police forces. All of these systems need to be maintained and further developed on a continuous basis. As a consequence, specialized private firms will be drawn ever deeper into the operational work of state security services, further increasing sociopolitical ambiguities.

During the golden years of globalization, liberals hoped that global technology norms and regulatory standards would increasingly converge. But while the technology innovation space increasingly expanded around the globe, alternative technology norms and regulatory standards began to emerge in the 21st century, based on a different vision of state-business relationship. China heavily invested in so-called national technology champions, installed a "Civil Military Fusion" mechanism (Bitzinger 2021), and began to actively influence the development of international technology standards (US-China Business Council 2020; Li and Chen 2021). As Western states increasingly perceived China as a geopolitical competitor, they began to set up foreign direct investment screening mechanisms and broadened their dual-use export control systems with the aim of limiting China's access to the West's technology innovation space. In parallel, they began to look for new ways of how best to secure their states' - and especially their security services' - access to their national technology base. As Danny Steed (2022) argues in his chapter, the Snowden revelations substantiated the extent to which Western technology firms shared data with the US state in the name of national and international security.

The upshot of these developments is that the governance space around emerging technologies has become increasingly fragmented and plagued by socio-technical and sociopolitical ambiguity. Cyberspace was originally created as a politically open space with governance structures limited to its technical architecture. As Steed notes in his chapter, the existing sovereignty gap in cyberspace "is not the source of fragmentation, it is the growing [geopolitical] competition to fill the gap that is". The same applies to the growing interconnectedness between cyberspace and outer space, as Johan Eriksson and Giampiero Giacomello (2022) show in their chapter. Private actors increasingly drive the space technology innovation process, as an increasing number of (cyber) infrastructures depends on space-based satellite services. Yet at the same time, state militarization and politicization of outer space accelerates, as an ever-growing number of states use satellite technologies to modernize their security services. The coming together of these two trends creates new vulnerabilities and new types of treats (e.g. anti-satellite weapons, space debris). At the same time, it increases political fragmentation. The diversification of private and state actors raises the old question with new urgency if and how publicprivate partnerships can secure technological reliability and long-term investment.

\section{Strategic stability under multidimensional uncertainty}

The assumed revolutionary potential of cyberspace, Miguel A. Gomez and Christopher Whyte (2022) note in their chapter, was the product of the twin 
uncertainties about the scope and tempo of the technical innovation and the related social and political responses. As new technological possibilities emerged, politics began to catch up in a process of sociopolitical normalization. As a consequence, state behavior evolved over time. In the absence of a demonstrable strategic utility of cyber operations and a strategic context characterized by a puzzling co-existence of restraint at the high end of conflict and persistent low-level cyber conflict, key states started to increasingly move away from deterrence to cyber conflict management. In 2018, the United States issued a new cyber strategy signaling a shift to persistent engagement and defend forward. The logic of the new approach emphasized that the characteristics of the operational environment in cyberspace - a space of constant contact - demand a continuing engagement and degradation of adversarial cyber capabilities and operations wherever they were found (US Cyber Command 2018; US Department of Defense 2019).

This shift away from deterrence might be premature and underestimate the potential of (unintended) escalation, Gomez and Whyte argue. Moreover, it is still unclear why states invest substantial technical, financial, and organizational resources in using the domain offensively if cyber operations are indeed of limited strategic utility only. Within this context, the chapters in this volume focus on three interconnected aspects of upholding strategic stability under multidimensional uncertainty. A first subsection concentrates on the micro-dynamics of decision-making that might drive escalation under uncertainty and ambiguity. The focus here is on how prior beliefs and cognitive biases might influence the response decisions of elite stakeholders in varying national strategic cultures. A second subsection deals with the ambiguities of attribution as a precondition for a credible deterrence threat. The focus here is on how policymakers perceive cyberspace as a completely human-built domain and how this translates into political apprehension about the applicability of deterrence in cyberspace. A third subsection analyzes the growing role of intelligence in cyberspace. The focus here is on how the digitalization of intelligence changed its strategic and operational role and what (un)intentional consequences this had for cyber insecurity, on the one hand, and for great powers' views on (un)acceptable behavior of intelligence services in cyberspace, on the other.

\section{Escalation: The micro-dynamics of decision- making in varying sociopolitical contexts}

Precisely because it is difficult to control the strategic effects of cyber (influence) operations, more research is needed on the micro-dynamics of decision-making that may drive unintended escalation. Contributing to the behavioral turn in cyber security research, Miguel A. Gomez and Christopher Whyte (2022) investigate the effects of uncertainty on judgment in the context of (crisis) decision-making under cyberattack. In such situations, the ambiguity of diffuse actors and malicious actions increases the uncertainty of decision-makers about both the intent behind and the consequences of cyber (influence) operations. The authors use war gaming as a pseudo-experimental method to determine if and how decision-makers 
use well-known heuristic mechanisms such as prior beliefs and analogical reasoning to discern intent and consequences behind cyber operations. The authors find distinct evidence in support of the notion that decision-makers, when faced with digital insecurity and the use of adversarial cyber operations, fall back on noncyber situations to make their task simpler.

The degree to which heuristic shortcuts interfere with objectivity and results in more or less severe responses depends on distinct national (strategic) cultures. Gomez and Whyte discuss evidence of cross-national cultural variations influencing the response decision among elite stakeholders. The socio-institutional correlates of civil-military relations in a given democracy stand out to have a unique impact on decision-making processes. Based on their observations from cross-national war games, they conclude that the interaction between the microfoundations of decision-making in a given cultural and institutional setting "might ultimately have some effect on the strategic calculations states make around signaling and adversary behavior" (Gomez and Whyte 2022: 125). The fact that unintended escalation due to prior beliefs, cognitive biases of decision-makers, and/ or bureaucratic politics cannot be excluded in strategic context characterized by uncertainty and ambiguity highlights the advantage of deterrence as a conflict management tool: As a theory of interdependent decision-making, it might prevent militarization and escalation (Schelling 1966).

\section{Deterrence: The ambiguity of attribution in the context of cyber conflict management}

Over the years there has been considerable work invested at the science-policy interface in adapting deterrence to the ambiguous context of cyberspace. The scope of the practical applicability of the tenets of deterrence to cyberspace is considerably more limited than in more traditional conventional and nuclear deterrence settings (Soesanto and Smeets 2020). At the same time, deterrence attempts in cybersecurity and cyber defense span a wide spectrum of threats, including cybercrime, cyber espionage, and operational cyberattack.

From a conceptual point of view, the attention at the lower end of conflict shifted to criminological conceptions of deterrence and from punishment to denial mechanisms converging on target hardening through cyber resilience (Wenger and Wilner 2021). In such settings, though, deterrence approaches are typically integrated with other coercive and non-coercive tools into a broader conflict management strategy. Conversely, at the higher end of conflict the attention of strategists has shifted to the concept of cross domain deterrence (Lindsay and Gartzke 2019). The focus here is on adversaries that apply ambiguous "gray zone" strategies that integrate military and non-military coercive instruments while evading attribution. Cross domain deterrence tends to include both positive inducements and negative threats and brings the concept of deterrence "back to the broader coercive diplomacy literature from which it originally emerged" (Sweijs and Zilinick 2021: 152).

In his chapter on the limited reliance of Israel on cyber deterrence, Amir Lupovici (2022) explores how new digital technologies enter into doctrine and 
strategy. Acknowledging the methodological difficulties of studying cyber deterrence, he deliberately shifts the focus from studying what makes deterrence effective in a given strategic context to analyzing how the cyber domain is embedded in Israel's strategic culture and identity. From such a viewpoint, Lupovici argues, it is puzzling that Israel has so far not developed a clear cyber deterrence strategy, given the prominent role deterrence has played in Israel's strategy and the country's "deterrer identity" (Lupovici 2016). Israeli policymakers, he concludes, seem to recognize the uncertainty and ambiguity involved in establishing a deterrence balance in cyberspace and consequently shy away from formulating a declaratory cyber deterrence strategy.

From an operational point of view, Lupovici (2022) argues, Israel's repeated use of offensive cyber operations against the Iranian and Syrian nuclear programs have been interpreted by some experts as attempts to establish cumulative deterrence through the actual use of force, a concept which is deeply ingrained in Israeli strategic culture (Adamski 2021). Yet the effectiveness of such a strategy remains in dispute, Lupovici insists, and whatever deterrent threat might get through to the adversary is communicated in an indirect and implicit way only. From a conceptual perspective, the US strategy of persistent engagement and defend forward seems to share some of the tenets of the Israeli concept of cumulative deterrence (Tor 2015; Kello 2017). Yet the concept of cumulative deterrence was customarily rejected by most US strategists and policymakers, since in the context of nuclear deterrence the use of force was seen as a symptom of deterrence failure, signalizing a shift from a policy of influence to a policy of control (Adamsky 2021).

It is quite telling that two of the leaders in thinking about and in practicing deterrence in their different strategic contexts have come to accept the limits of deterrence in cyberspace. The way US and Israeli policymakers and strategist conceptualize the cyber domain - as an operating environment with a high degree of technical interconnectedness (increasing uncertainty) and constant political contestation (increasing ambiguity) - seems to be part of the explanation why they, respectively, moved away from cyber deterrence (United States) and never declared a clear deterrence strategy (Israel). Although cyberspace is conceptualized as the fifth domain of warfare, its structural characteristics differ from the other four domains. Cyberspace is completely human-built, shaped by technology companies, and operating in it will always be hard and only partially under control of any one actor (Seebeck 2019). Precisely because cyberspace is completely designed by humans, states can shape it according to their interest. Yet as in cryptology they must cooperate to compete and accept the constraint of a cooperatively produced network of networks.

The economic and political logic of cyberspace as something completely designed by humans might explain why states seem to perceive cyberspace as a domain of intelligence rather than warfare. As discussed above, the fact that setting up cyber exploitation is more expensive than countering released exploitation translates into an incentive to keep the target at risk. From a political point of view, transparent attribution as a precondition of a credible deterrence threat is 
difficult. Relating an intrusion set to a politically responsible party, Kuerbis et al. (2022) argue, remains challenging because it includes a judgment about the relation between victim and adversary. As such, it should be interpreted as "a product more of political science or intelligence studies than computer science" (Kuerbis et al. 2022: 222).

\section{Intelligence: The growing operational role of intelligence as a source of cyber insecurity}

In the context of the multidimensional uncertainty prevailing in cyberspace, intelligence agencies have turned into one of the most dominant actors in this human-built domain (Buchanan 2020; Egloff 2022). Their role in cyber conflict is a paradox and highly ambiguous one: They represent both the biggest threat and the most capable provider of security and safety. Such an outcome is not without irony, because the technical transformation from an analog to a digital world exposed them to a mortal threat: going dark. In his chapter on the consequences of the digital disruption of the second oldest profession, Danny Steed (2022) discusses how US and British intelligence "mastered the internet". In the process, he concludes, they not only transformed their role in security and defense, but unintentionally exacerbated cyber insecurity.

As global information flows began moving into fiber optics, US and British intelligence adapted their skillset to one that could penetrate digital codes and infrastructure (Buchanan 2020). Exploiting the sovereign geographic access to the submarine cables through which the bulk of the internet traffic traversed, was a key factor for success, as was a close partnership with numerous technology companies that facilitated access and sharing of meta-data. The solution to the old intelligence adage - to find the needle in the haystack - was found in technical innovation, as Steed explains: The two intelligence services temporarily collected the whole haystack in a buffer system, which allowed them to sort out relevant information and meta-data via automated analysis. This unique access to large volumes of internet traffic created intelligence dependencies even among close allies, as Stefan Steiger (2022) shows in his chapter on Germany's cyber security politics. Once the Snowden leaks highlighted that foreign intelligence was an accepted state practice even among allies, the German government in a partnership with Brazil invested into a new submarine cable across the Atlantic.

Intelligence services are the most purposefully ambiguous tools of statecraft. The legal ambiguity of intelligence in domestic and international law was for a long time based on the reciprocal assumption of great powers that intelligence services would help decision-makers guard against a military fait accompli and uphold strategic stability. The purpose of the limited intrusion into the sovereign affairs of another state was to provide enough transparency to avoid rapid escalation. In the context of their digital transformation, Danny Steed (2022) contends, their strategic relevance increased considerably. At the same time, their operational focus increasingly shifted from assessing uncertainty to eliminating uncertainty. The shift to a more operational role needs to be seen in the context of 
a unipolar world, in which the management of transnationally networked threats - terrorism, extremism, organized crime, cyberattacks, WMD proliferation dominated Western policy and strategy. In the post-9/11 context, operational intelligence and close (bilateral) cooperation among asymmetrically dependent intelligence services played a preeminent role in the global management of the then dominant security challenges.

With the return of geopolitical rivalry between great powers and in the context of the pro-active use of intelligence and cyber (influence) operations by rising powers, international disagreement about what should be considered acceptable use of espionage began to multiply, as noted above in section one. From the perspective of great power politics, it seems essential that states sort out the difference between mutually acceptable espionage in support of strategic stability and inacceptable meddling in the internal political and economic affairs of another state. The 2015 mutual agreement between China and the United States, in which both states committed to not conducting or supporting economic cyber espionage (Baezner and Robin 2017), and the recent agreement between Biden and Putin to conduct "experts-level talks" on red lines for cyberattacks on "critical" sectors (Hirsh 2021), might be read as early beginnings of a long haul toward a tacit understanding of acceptable behavior of intelligence services in cyberspace.

It seems highly unlikely, however, that talks at the diplomatic level will result in a breakthrough any time soon. For this to happen, the differences of acceptable surveillance at the domestic level are simply too big. Societies need to know how their intelligence services work in cyberspace, because their tools and practices set practical norms with far-reaching effects on state, society and economy (Georgieva 2020). For authoritarian states, the priority is to control citizens' access to information, while for democracies the priority is to protect individual privacy and intellectual property rights. Questions about privacy, security, information are at the heart of the political struggle about cyber security and this makes the quest for global norms of responsible behavior in cyberspace a slow and difficult one.

The manner in which intelligence services mastered the internet, Danny Steed (2022) convincingly argues, created additional socio-technical uncertainty and exacerbated the cyber security challenge. Digitalization made intelligence more visible, because unlike in an analog world spies now worked within the same digital infrastructure as all other social, economic, and political actors. As a consequence, intelligence intrusion could be exposed much faster than before, which made intelligence far more visible. When whistle-blowers brought their activities into the spotlight, domestic and international political contestation about their role multiplied. As a corollary of the exposure of intelligence methods, intelligences services turned into inadvertent proliferators of malicious code and zeroday exploits. As a consequence, more people were enabled to use intelligence tools for malicious purposes - compared to intelligence services, with no oversight whatsoever. Some of these tools were later deployed in two global malware attacks - WannaCry and NotPetya - further increasing the ambiguity of action in cyberspace and the uncertainties of attribution. 


\section{Emerging governance responses: Policy coordination and norms formation}

The socio-technical expansion of cyberspace is led by private technology firms, yet state actors shape the tighter coupling of technical systems with sociopolitical institutions. This in turn means that governments share the responsibility to secure cyberspace with actors from the economy and society. In the process of these socio-technical and sociopolitical transformations, emerging cyber governance responses unfold in an increasingly transnational and trans-sectoral policy space. The vision of a wireless, satellite-based internet accessible to everyone propagated by private business actors and the parallel reality of state actors that are increasingly politicizing and militarizing outer space is set to further expand cyberspace as a transnational policy space. As a network of interdependent information technology infrastructures, cyberspace is connected across state borders and through global satellite-based communications services. At the same time, cyberspace as a trans-sectoral policy space also expands rapidly. The tighter coupling of ever more socio-technical systems increases the interconnectedness of cyberspace. As a consequence, cyber security affects a rapidly growing number of different policy fields.

The key governance challenge in cyberspace is how to overcome fragmentation of authority and accountability. Within the context of a trans-sectoral and transnational policy space, the chapters in this volume highlight three aspects of the ongoing re-negotiation processes among state, society, and economy about their roles and responsibilities in cyberspace. A first subsection deals with the significant expansion of state responsibilities in cyberspace over the past decades. The focus is on how state actors fine-tuned their multidimensional role across different policy fields in a process that was influenced by distinct patterns of interaction between domestic contestation and international orientation and dependence. A second subsection concentrates on the increasingly prominent role of private and civil society actors in the search for new forms of transnational governance in cyberspace. The focus here is on the norm-based activities of big tech companies, on the one hand, and a series of proposals for a global platform for transnational attribution, on the other. A third subsection brings the attention back to state actors, shedding light on the critical role of intelligence services in (in)securing cyberspace. As long as great powers disagree about what constitutes acceptable behavior of intelligence services in cyberspace, the systemic levels of insecurity in cyberspace will likely not materially decrease.

\section{Growing role of governments: Shifting patterns of domestic and international governance}

The tech pioneers had built the internet based on the vision of an open technical governance infrastructure with minimal involvement of government. Yet as cyberattacks were becoming more persistent, more targeted, more expensive, and more disruptive, governments began to significantly expand their roles and 
responsibilities in cyberspace. Ever since states find themselves in the midst of two interlinked re-negotiation processes of their roles and responsibilities in (securing) cyberspace. While at the level of domestic politics they renegotiate their role in securing cyberspace as a shared responsibility with society and industry, at the level of international politics they renegotiate the patterns of international governance with states, private and civil actors.

In his chapter, Stefan Steiger (2022) analyzes how Germany's cyber security policies evolved over time, shaped by the complex interactions between domestic and international negotiation processes. He employs a role theoretical two-level game to analyze how domestic and international factors influenced the development of German cyber security policy. Isolating four interconnected policy domains - critical infrastructure protection (CIP); law enforcement; intelligence services; military - he discusses how varyingly fragmented national and international actors reached four distinct, but still connected policy outcomes. The CIP and law enforcement domains of German cyber security policy comprise the most distinct international and regional cooperation patterns, Steiger concludes. In the CIP domain, domestic CIP policies emerged first, based on a model of public-private partnerships that delegated the primary responsibility for cyber security to the private sector. Over time, however, the federal government strengthened its supervisory role considerably. The German government promoted the protection of critical infrastructures also internationally, primarily in the EU and the OSCE, reflecting the physical interconnectedness of critical infrastructure across borders. In the domain of law enforcement, EU members successfully harmonized criminal law, without however weakening the central authority and sovereignty of the (German) state.

The intelligence and military domains of German cyber security policy remain intergovernmental policy domains, in which the German government accepted no self-binding regulations. The intelligence domain exhibits the most paradoxical interaction patterns between national and international re-negotiation processes, Steiger notes. On the one hand, Germany's early call for international restraint in cyberspace was facilitated by the intelligence dependence on the United States. Once the Snowden revelations showed that digital surveillance was an accepted state practice even among allies, the role of intelligence was hotly contested at the domestic level. As a consequence, Germany expanded the legal basis for foreign surveillance, began to stockpile zero-day exploits, and expanded its access to the transatlantic internet traffic. In parallel, this expanded foreign mandate of German intelligence was balanced with stronger domestic control mechanisms and special protection rights for German and EU citizen. In the military domain too, Germany, because of its commitment to NATO, began to move away from international restraint, established a cyber-command, and prepared for the use of offensive cyber capabilities.

Chapters 11 and 12 in this volume offer two additional case studies discussing the cyber security policies of two states that are located at the periphery of Europe and want to move closer to Western institution. The evolution of their cyber security policies too is characterized by distinct patterns of interaction between domestic institutional transformation and international orientation and 
dependence. We have already discussed the case of Ukraine above. Ukraine represents the extreme case of a small state with weak cyber capacity that sits on the geopolitical fault lines between Russia and the West. Exposed to persistent Russian cyberattacks and massive information operations, the country recently lived through a domestic revolution linked to an abrupt reorientation of its foreign policy alignment from Russia to Europe and the West.

In his chapter, Aaron Brantly (2022) shows that the pattern of domestic contestation and international reorientation resulted in a fairly successful response of the country to Russia's information warfare, aimed at undermining the social and political fabric of Ukraine. He explains this as the result of a combination of a series of top-down government initiatives - including restrictive moves against Russian-dominated web platforms and broadcast channels and the introduction of a Ministry of Information Policy - with a series of bottom-up initiatives by journalist and externally sponsored NGOs - focusing on fact checking, disclosure of foreign propaganda, and the training of journalists and civil society. Less successful, however, were the country's efforts to increase its resilience against cyberattacks. Although the country aligned the legal and organizational foundations of its cyber security policies with EU and NATO standards, the new cyber security structures are not yet functional on their own, Brantly concludes. He points to the dominance of old bureaucratic cultures - especially in the security services - and dependence on external assistance and funding as the two main reasons for weak policy implementation.

Albania represents another interesting case of a small state with weak cyber capabilities that is transforming toward democracy and wants to move closer to Western institutions. In his chapter, Islam Jusufi (2022) discusses how both the cyber threat frames and the policy responses visible in Albania's policy documents diffused from the international level - especially from US, UK, EU, and NATO sources. This policy diffusion process to the national level had two notable consequences, the author argues: First, cyber security was preemptively upgraded to a national threat level, i.e. not in response to national incidents. Second, the new policy introduced the concept of multi-stakeholder governance that represented a shift from Albania's traditional state-centered governance model. In combination, these two developments resulted in a somewhat paradoxical outcome: While the dependence of a technologically weak state on foreign actors increased, the introduction of new international policy concepts augmented the fragmentation of domestic authority in cyber security. Moreover, this outcome highlights a certain time-inconsistency problem in international policy coordination. It is not without irony that Western states in parallel began to reclaim authority and sovereignty in certain policy domains - as demonstrated in the German case above - and expanded the protector role of the government in cyberspace.

\section{Toward new forms of transnational governance: Norms and institutions}

The search for new forms of transnational governance reflects a realization that digital technologies and the services they provide are increasingly connected 
across state borders and into outer space. Why do private and civil society actors play an increasingly prominent role in the development of norms and institutions that aim to regulate human behavior in cyberspace? First, cyber norms and institutions remain contested at the level of international politics. The inability of states to make progress in the direction of a common understanding of cyber norms, especially at the United Nations, provided the context for a growing engagement of non-state actors. Second, a series of large-scale data breaches and malware strikes undermined social trust - a critical success factor for the business models of transnationally operating tech firms - in the socio-technical systems that constitute cyberspace. Third, the mostly private creators of cyber space possess key engineering expertise that is essential to ensure that new governance approaches are anchored in a tacit understanding of research and development and broader business practices. In turn, civil society has the potential to provide additional benefits in terms of transparency, privacy, and equality.

In her chapter, Jacqueline Eggenschwiler (2022) evaluates the norm-based activities of big tech companies, including Kaspersky Lab, Siemens, Telefónica, and Microsoft. She introduces norms approaches as appropriate regulatory approaches to tackle the contextual ambiguities of fast-moving environments, which preempt costly - and from the viewpoint of tech firms unwanted - changes to legal frameworks. With their voluntary engagement in support of the development of cyber security norms of responsible behavior, technology firms aim to define responsible product development and engineering practices and establish trust in social interactions enabled by digital technologies. The norm-based activities of big tech have been partially successful insofar as they have converged on a number of widely shared normative ideas and design principles and injected these ideas and principles into a number of regional and international political processes. The procedural effects of a greater inclusion of private and civil society actors in norm development processes will likely be enduring, Jacqueline Eggenschwiler concludes. Yet big tech's push for cyber security norms has not resulted in a substantial reduction of cyber insecurity.

Not only the development of cyber security norms will be a long process, the same is true for the institutionalization of a recognized transnational attribution process, Brenden Kuerbis, Farzaneh Badiei, Karl Grindal, and Milton Mueller (2022) argue in their chapter. Cyber attribution as a socio-technical and highly interdisciplinary endeavor is a precondition for the deterrence of cyberattacks and a precursor for stable social relations in cyberspace. The current attribution claims of threat intelligence firms and national security services are however often based on limited evidence and the reputation of the attributing actor, and, as a consequence, lack transparency and credibility. However, new advances in attribution that combine better algorithm-driven technical attribution with better understanding of the institutional condition under which attribution might occur, Kuerbis et al. note, may in the future improve the baseline for institutionalizing transnational attribution.

The chapter discusses various proposals from private actors and academic institutions on how a global platform for transnational attribution could be set up 
and what the scope of its activities should be. The following two major challenges on the way toward implementation stand out in most of them: A first key question is how to ensure the technical independence of such a platform and the professionalism of the participants. There is still a lot of research needed to define the scientific and methodological standards, including transparency, reproducibility, and falsifiability, of the practice of attribution. In addition, it remains unclear why private tech firms with advanced forensic capabilities would participate in such a platform. A second key question is how to guarantee the judicial independence of such a platform and what governance form would be effective in aligning the participants' incentives. The spectrum of conceivable solutions ranges from hierarchically organized institutions to loosely organized forms of networked governance. In the final analysis, however, the success of private and civil sector-driven cyber security norms processes as well as of initiatives aimed at the institutionalization of transnational attribution critically depend on the political will of state actors, especially great powers, to agree upon norms of responsible behavior as the ultimate enforcer.

\section{Great powers as ultimate enforcers: Re-negotiation, the ambiguous norms of espionage}

States cannot govern cyberspace on their own, they need to integrate economic and social actors into a wider cyber security governance framework. Yet no stable cyber security governance framework will evolve without greater convergence among great powers on responsible state behavior as ultimate enforcers. It is therefore vital for non-state and state actors to work closer together and aid one another in their behavior-shaping efforts in order to decrease the systematic levels of cyber insecurity, Jacqueline Eggenschwiler (2022) argues in her chapter. As long as emerging (information) technologies are perceived as a geopolitical battleground, limited progress will be possible. States need to negotiate a tacit understanding about what constitutes a mutually acceptable balance between restraint in and exploitation of cyberspace. As discussed above, a critical component of such an understanding is linked to the behavior of state intelligence services in the digital domain. The great power's views on what forms of espionage and interference in the political processes and socioeconomic activities of other societies through cyberspace are acceptable need to converge before the systemic levels of cyber insecurity will materially decrease.

\section{Conclusion}

The chapters in this book discussed the ambiguity of current cyber security politics in an uncertain context characterized by rapid socio-technical transformation and increasing fragmentation of political authority. In this concluding chapter, we highlighted four key debates in current thinking about cyber security, all of them linked to the interplay between technological possibilities and political choices in cyberspace. An analytical perspective that emphasizes the co-constitution 
and co-dependency of technology and politics provides an especially productive lens for studying the complexities and paradoxes of cyber security politics. The key reason for this is found in the nature of cyberspace as a domain completely designed and built by humans - with a high degree of technical interconnectedness and constant political contestation. As a consequence, state, economy and society must cooperate to compete in cyberspace and accept the constraints of a cooperatively produced network of networks.

A key insight that such an analytical perspective offers is that both evolving cyber threat narratives and emerging cyber governance responses are co-produced by state and non-state actors in a rapidly changing trans-sectoral and transnational policy space. Emerging cyber threats are co-constituted by the micro-politics of technology design decisions in competitive global markets, the meso-politics of technology norms choices in competitive regulatory environments, and the macro-politics of great powers that act strategically in a competitive international system. Within this broader context, the chapters in this book highlight a series of interaction mechanisms between technology and politics that influence cyber threat politics in different strategic contexts: Tech race dynamics around emerging dual-use technologies clearly leave a mark in the national threat politics of great powers. Actors in "gray zone" conflicts attempt to manipulate the opaqueness of cyber (influence) operations. And in democracies policymakers are increasingly concerned about the asymmetrical vulnerability of their socio-technical public sphere to foreign disinformation and cyber influence campaigns.

That cyber threat perceptions are co-constituted by technology and politics also means that their realization is not predetermined. Both state and non-state actors can contribute to a decrease of the level of insecurity in cyberspace. States need to establish red lines, uphold strategic stability, and develop norms of responsible state behavior in cyberspace. Actors from society and economy need to develop norms of responsible behavior for the creators and users of emerging technologies as the bedrock of societies' trust in socio-technical systems. Yet the effectiveness of their individual responses to cyber threats depends on their mutual interplay. States and societal actors need to negotiate how public authority is exercised in cyberspace. A stable governance framework for cyber security can only emerge if great powers develop a tacit understanding on what represents a responsible use of cyber operations in state interactions, and societal actors successfully navigate the normative space around technology, information, privacy, and security.

Researchers can contribute to the search for a functioning governance framework: They can highlight the less visible actors in cyberspace, design and evaluate new socio-technical institutions to secure cyberspace or monitor, and analyze publicly available data about cyber operations. A key conceptual challenge for cyber security research is linked to the integration of theoretical knowledge from different disciplines that allows to analyze the many interactions between the international dimension of cyber security politics and the broader dimension of cyber security politics (Dunn Cavelty and Wenger 2019). Those who study the former tend to build on approaches from IR, security, and intelligence studies, but increasingly recognize broader contributions from critical security studies and 
practice theory. Those who study the latter, leverage an even broader array of theoretical perspectives including approaches from IPE, governance studies, and the IR norms literature.

A practical challenge is how to overcome the dominance of Western perspectives both in politics as well as in academia. We tend to see only the peak of an iceberg of malicious activities in cyberspace that is linked to the political and economic interests of Western states and threat intelligence firms. The empirical focus of most chapters in this book is informed by the geostrategic rivalry between Western democracies and Russia and China as their main authoritarian contender. It is this strategic context and the differences in the domestic institutional setup of the leading great powers that guide large parts of the analyses of cyber conflict in this volume. Yet at the same time, individual chapters point to interesting variances in the cyber security policies among traditional (United States, Israel) and aspiring (Albania, Ukraine) democracies, on the one hand, and to the important role of cross-national cultural variations in cyber decision-making, on the other. Cyber security is increasingly negotiated at the global level and this is why we need to better understand how different regions and cultures think about the interplay of technology and politics in cyberspace.

\section{Bibliography}

All links checked on September 3, 2021.

Adamsky, D. D. (2021). Deterrence by Denial in Israeli Strategic Thinking. In A. Wenger and A. Wilner (eds), Deterrence by Denial: Theory and Practice. Amherst, NY: Cambria Press, 163-190.

Baezner, M. (2018). Hotspot Analysis: Synthesis 2017: Cyber-Conflicts in Perspective. Zurich: Center for Security Studies (CSS).

Baezner, M., and Cordey, S. (2022). Cyber in the Grey Zone: Influence Operations and other Conflict Trends. In M. Dunn Cavelty and A. Wenger (eds), Cyber Security: Socio-Technological Uncertainty and Political Fragmentation. London: Routledge, pp. 17-31.

Baezner, M., and Robin, P. (2017). Hotspot Analysis: Strategic stability between Great Powers: the Sino-American Cyber Agreement. Zurich: Center for Security Studies (CSS).

Bitzinger, R. A. (2021). China's Shift from Civil-Military Integration to Military-Civil Fusion. Asia Policy, 28(1): 5-24. https://doi.org/10.1353/asp.2021.0001.

Bonfanti, M. E. (2022). Artificial Intelligence and the Offence-Defence Balance in Cyber Security. In M. Dunn Cavelty and A. Wenger (eds), Cyber Security: Socio-Technological Uncertainty and Political Fragmentation. London: Routledge, pp. 64-79.

Borghard, E. D., and Lonergan, S. W. (2017). The Logic of Coercion in Cyberspace. Security Studies, 26(13): 452-458.

Brantly, A. (2022). Battling the Bear: Ukraine's Approach to National Cyber and Information Security. In M. Dunn Cavelty and A. Wenger (eds), Cyber Security: SocioTechnological Uncertainty and Political Fragmentation. London: Routledge, pp. 157-171.

Buchanan, B. (2020). The Hacker and the State: Cyber Attacks and the New Normal of Geopolitics. Harvard: Harvard University Press. 
Cavaiola, L. J., Gompert, D. C., and Libicki, M. (2015). Cyber House Rules: On War, Retaliation and Escalation. Survival, 57(1): 81-104.

Chesney, R., and Smeets, M. (2020). Roundtable: The Dynamics of Cyber Conflict and Competition. Texas National Security Review, 3(4): 4-7. https://doi.org/10.26153/tsw /10964.

Chesney, R., and Smeets, M. (forthcoming). Deter, Disrupt or Deceive: Assessing Cyber Conflict as an Intelligence Contest. Washington: Georgetown University Press.

Clarke, R. A., and Knake, R. K. (2010). Cyber War. New York: Ecco.

Devanny, J. (2021). 'Madman Theory' or 'Persistent Engagement'? The Coherence of US Cyber Strategy under Trump. Journal of Applied Security Research. Retrieved August 19, 2021, from: https://www.tandfonline.com/doi/abs/10.1080/19361610.2021 .1872359 .

Dewar, R. S. (ed.). (2018). National Cybersecurity and Cyberdefense Policy Snapshots. Zurich: Center for Security Studies (CSS).

Dunn Cavelty, M. (2008). Cyber Security and Threat Politics: US Efforts to Secure the Information Age. London: Routledge.

Dunn Cavelty, M. (2015). The Normalization of Cyber-International Relations. In O. Thränert and M. Zapfe (eds), Strategic Trends 2015: Key Developments in Global Affairs. Zurich: Center for Security Studies (CSS), pp. 81-98.

Dunn Cavelty, M., and Egloff, F. J. (2019). The Politics of Cybersecurity: Balancing Different Roles of the State. St Antony's International Review, 15(1): 37-57.

Dunn Cavelty, M., and Wenger, A. (2019). Cybersecurity Meets Security Politics: Complex Technology, Fragmented Politics, and Networked Science. Contemporary Security Policy, 41(1): 5-32.

Dunn Cavelty, M., and Wenger, A. (2022). Introduction: Cyber Security between SocioTechnological Uncertainty and Political Fragmentation. In M. Dunn Cavelty and A. Wenger (eds), Cyber Security: Socio-Technological Uncertainty and Political Fragmentation. London: Routledge, pp. 1-13.

Eggenschwiler, J. (2022). Big Tech's Push for Norms to Tackle Uncertainty in Cyberspace. In M. Dunn Cavelty and A. Wenger (eds), Cyber Security: Socio-Technological Uncertainty and Political Fragmentation. London: Routledge, pp. 186-204.

Egloff, F. J. (2020a). Contested Public Attributions of Cyber Incidents and the Role of Academia. Contemporary Security Policy, 41(1): 55-81. https://doi.org/10.1080 /13523260.2019.1677324.

Egloff, F. J. (2020b). Public Attribution of Cyber Intrusions. Journal of Cybersecurity, 6(1): 1-12. https://doi.org/10.1093/cybsec/tyaa012.

Egloff, F. J. (2022). Semi-State Actors in Cybersecurity. Oxford: Oxford University Press.

Egloff, F. J., and Dunn Cavelty, M. (2021). Attribution and Knowledge Creation Assemblages in Cybersecurity Politics. Journal of Cybersecurity, 7(1): tyab002. https:// doi.org/10.1093/cybsec/tyab002.

Egloff, F. J., and Wenger, A. (2019). Public Attribution of Cyber Incidents. In F. Merz (ed.). CSS Analyses in Security Policy, 244. Zurich: Center for Security Studies (CSS), pp. $1-4$.

Eriksson, J., and Giacomello, G. (2022). Cyberspace in Space: Fragmentation, Vulnerability, and Uncertainty. In M. Dunn Cavelty and A. Wenger (eds), Cyber Security: SocioTechnological Uncertainty and Political Fragmentation. London: Routledge, pp. 95-107. 
Fischer, S.-C. (2021). The Mobilization of Commercial Technology Companies: Explaining the Pursuit of U.S. Technological Superiority vis-à-vis China in a Private Sector-Driven and Globalized Innovation System. Unpublished PhD Manuscript. Zurich: Center for Security Studies (CSS).

Fischer, S.-C., and Wenger, A. (2019). A Politically Neutral Hub for Basic AI Research. CSS Policy Perspectives, 7(2). Zurich: Center for Security Studies (CSS).

Fischer, S.-C., and Wenger, A. (2021). Artificial Intelligence, Forward-Looking Governance and the Future of Security. Swiss Political Science Review, 27(1): 170-179. https://doi .org/10.1111/spsr.12439.

Gartzke, E. (2013). The Myth of Cyberwar. Bringing War in Cyberspace Back Down to Earth. International Security, 38: 41-73. https://doi.org/10.1162/ISEC_a_00136.

Georgieva, I. (2020). The Unexpected Norm-Setters: Intelligence Agencies in Cyberspace. Contemporary Security Policy, 41: 33-54. https://doi.org/10.1080/13523260.2019 1677389.

Gomez, M. A., and Whyte, C. (2022). Cyber Uncertainties: Observations from CrossNational Wargames. In M. Dunn Cavelty and A. Wenger (eds), Cyber Security: Socio-Technological Uncertainty and Political Fragmentation. London: Routledge, pp. 111-127.

Harknett, R. J., and Smeets, M. (2020). Cyber Campaigns and Strategic Outcomes. Journal of Strategic Studies, Ahead of Print: 1-34. https://doi.org/10.1080/01402390.2020 .1732354 .

Haunschild, J., Kaufhold, M.-A., and Reuter, C. (2022). Cultural Violence and Peace in Social Media: Technical and Social Interventions. In M. Dunn Cavelty and A. Wenger (eds), Cyber Security: Socio-Technological Uncertainty and Political Fragmentation. London: Routledge, pp. 48-63.

Healey, J. (2019). The Implications of Persistent (and Permanent) Engagement in Cyberspace. Journal of Cybersecurity, 5(1): tyz008. https://doi.org/10.1093/cybsec/ tyz008.

Healey, J., and Jervis, R. (2020). The Escalation Inversion and Other Oddities of Situational Cyber Stability. Texas National Security Review, 3(4): 30-53. https://doi.org/10.26153 /tsw/10962.

Hirsh, M. (2021, July 8). Putin Is Testing Biden's Cyber Resolve. Foreign Policy. Retrieved August 19, 2021, from: https://foreignpolicy.com/2021/07/08/putin-biden -cyber-security-attacks-ransomeware/.

Jervis, R. (1978). Cooperation under the Security Dilemma. World Politics, 30(2): 167-214. Jusufi, I. (2022). Uncertainty, International Obligations, Fragmentation and Sovereignty: Cyber Security in Albania. In M. Dunn Cavelty and A. Wenger (eds), Cyber Security: Socio-Technological Uncertainty and Political Fragmentation. London: Routledge, pp. 172-185.

Kello, L. (2017). The Virtual Weapon and International Order. New Haven: Yale University Press.

Kostyuk, N., and Zhukov, Y. M. (2017). Invisible Digital Front: Can Cyber Attacks Shape Battlefield Events? Journal of Conflict Resolution, 63(2): 317-47.

Kuerbis, B., Badiei, F., Grindal, K., and Mueller, M. (2022). Understanding Transnational Cyber Attribution: Moving from 'Whodunit' to Who Did it. In M. Dunn Cavelty and A. Wenger (eds), Cyber Security: Socio-Technological Uncertainty and Political Fragmentation. London: Routledge, pp. 220-238.

Lewis, J. A. (2018). Rethinking Cyber Security: Strategy, Mass Effects, and States. Washington, DC: Center for Strategic and International Studies. 
Li, X., and Chen, D. (2021, April 15). Should the West Fear China's Increasing Role in Technical Standard Setting? The Diplomat. Retrieved from: https://thediplomat.com /2021/04/should-the-west-fear-chinas-increasing-role-in-technical-standard-setting/.

Lindsay, J. R. (2013). Stuxnet and the Limits of Cyber Warfare. Security Studies, 22(3): 365-404.

Lindsay, J. R. (2014/2015). The Impact of China on Cybersecurity: Fiction and Friction. International Security, 39(3): 7-47.

Lindsay, J. R. (2017). Restrained by Design: The Political Economy of Cybersecurity. Digital Policy, Regulation and Governance, 19: 493-514. https://doi.org/10.1108/ DPRG-05-2017-0023.

Lindsay, J. R. (2020). Cyber Conflict vs. Cyber Command: Hidden Dangers in the American Military Solution to a Large-Scale Intelligence Problem. Intelligent and National Security, 36(2): 260-278. https://doi.org/10.1080/02684527.2020.1840746.

Lindsay, J. R. (2022). Quantum Computer and Classical Politics: The Ambiguity of Cryptologic Advantage. In M. Dunn Cavelty and A. Wenger (eds), Cyber Security: Socio-Technological Uncertainty and Political Fragmentation. London: Routledge, pp. 80-94.

Lindsay, J. R., and Gartzke, E. (2019). Cross-Domain Deterrence: Strategy in an Era of Complexity. Oxford: Oxford University Press.

Lupovici, A. (2016). The Power of Deterrence. Emotions, Identity, and American and Israeli Wars of Resolve. Cambridge: Cambridge University Press.

Lupovici, A. (2021). The Dog that Did not Bark, the Dog that Did Bark, and the Dog that Should Have Barked: A Methodology for Cyber Deterrence Research. International Studies Review, viab032. https://doi.org/10.1093/isr/viab032.

Lupovici, A. (2022). Uncertainty and the Study of Cyber Deterrence: The Case of Israel's Limited Reliance on Cyber Deterrence. In M. Dunn Cavelty and A. Wenger (eds), Cyber Security: Socio-Technological Uncertainty and Political Fragmentation. London: Routledge, pp. 128-140.

Maschmeyer, L. (2021). The Subversive Trilemma: Why Cyber Operations Fall Short of Expectations. International Security, 46(2): 51-90.

Maschmeyer, L., Abrahams, A., Pomerantsev, P., and Yermolenko, V. (forthcoming). Donetsk Don't Tell. Hybrid War in Ukraine and the Limits of Digital Influence Operations.

Poznansky, M., and Perkoski, E. (2018). Rethinking Secrecy in Cyberspace: The Politics of Voluntary Attribution. Journal of Global Security Studies, 3(4): 402-416. https://doi .org/10.1093/jogss/ogy022.

Rid, T. (2012). Cyber War Will Not Take Place. Journal of Strategic Studies, 35(1): 5-32.

Rid, T., and Buchanan, B. (2015). Attributing Cyber Attacks. The Journal of Strategic Studies, 38(1-2): 4-37.

Rovner, J. (2019, September 16). Cyber War an Intelligence Contest. War on the Rocks. Retrieved August 19, 2021, from: https://warontherocks.com/2019/09/cyber-war-as-an -intelligence-contest/.

Schelling, T. C. (1966). Arms and Influence. New Haven: Yale University Press.

Schulze, M. (2020). Cyber in War: Assessing the Strategic, Tactical, and Operational Utility of Military Cyber Operations. In 2020 12th International Conference on Cyber Conflict. Retrieved August 19, 2021, from: https://www.ccdcoe.org/uploads/2020/05/ CyCon_2020_10_Schulze.pdf.

Schünemann, W. (2022). A Threat to Democracies? An Overview of Approaches to Measuring the Effects of Disinformation. In M. Dunn Cavelty and A. Wenger (eds), 
Cyber Security: Socio-Technological Uncertainty and Political Fragmentation. London: Routledge, pp. 32-47.

Seebeck, L. (2019, September 5). Why the Fifth Domain is Different. The Strategist, ASPI (Australian Stratic Policy Institute). Retrieved from: https://www.aspistrategist.org.au/ why-the-fifth-domain-is-different/.

Slayton, R. (2017). What is the Cyber Offense-Defense Balance? Conceptions, Causes, and Assessment. International Security, 41: 72-109. https://doi.org/10.1162/ISEC_a 00267.

Smeets, M. (2018). The Strategic Promise of Offensive Cyber Operations. Strategic Studies Quarterly, 12(3): 90-113.

Smeets, M. (2022). No Shortcuts: Why States Struggle to Develop a Military Cyber-Force. London: Hurst Publishers.

Soesanto, S., and Smeets, M. (2020). Cyber Deterrence: The Past, Present, and Future. In F. Osinga and T. Sweijs (eds), NL ARMS Netherlands Annual Review of Military Studies 2020. The Hague: T.M.C. Asser Press. https://doi.org/10.1007/978-94-6265-419-8_20.

Steed, D. (2022). Disrupting the Second Oldest Profession: The Impact of Cyber on Intelligence. In M. Dunn Cavelty and A. Wenger (eds), Cyber Security: SocioTechnological Uncertainty and Political Fragmentation. London: Routledge, pp. 205-219.

Steiger, S. (2022). Cyber Securities and Cyber Security Politics: Understanding Different Logics of German Cyber Security Policies. In M. Dunn Cavelty and A. Wenger (eds), Cyber Security: Socio-Technological Uncertainty and Political Fragmentation. London: Routledge, pp. 141-156.

Sweijs, T., and Zilincik, S. (2021). The Essence of Cross-Domain Deterrence. In F. Osinga and T. Sweijs (eds), NL ARMS Netherlands Annual Review of Military Studies 2020. The Hague: T.M.C. Asser Press. https://doi.org/10.1007/978-94-6265-419-8_8.

Tor, U. (2015). 'Cumulative Deterrence' as a New Paradigm for Cyber Deterrence. Journal of Strategic Studies, 40(1-2): 92-117.

US-China Business Council (USCBC). (2020). China in International Standards Setting. USCBC Recommendations for Constructive Participation. Retrieved from: https:// www.uschina.org/sites/default/files/china_in_international_standards_setting.pdf.

US Cyber Command. (2018). Achieve and Maintain Cyberspace Superiority: Command Vision for US Cyber Command. Retrieved from: https://www.cybercom.mil/Portals /56/Documents/USCYBERCOM\%20Vision\%20April\%202018.pdf?ver=2018-06-14 $-152556-010$.

US Department of Defense. (2019). Cyber Strategy 2019: Summary. Retrieved from: https://media.defense.gov/2018/Sep/18/2002041658/1/1/1/CYBER_STRATEGY SUMMARY_FINAL.PDF.

Valeriano, B., and Maness, R. C. (2015). Cyber War versus Cyber Realities: Cyber Conflict in the International System. Oxford: Oxford University Press.

Wenger, A., and Wilner, A. (eds). (2021). Deterrence by Denial: Theory and Practice. Amherst, NY: Cambria Press. 